\title{
Vaccine-induced ADCC-mediating antibodies target unique and overlapping envelope epitopes
}

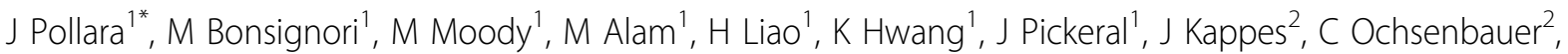 \\ K Soderberg ${ }^{1}$, TC Gurley ${ }^{1}$, DM Kozink' ${ }^{1}$ DJ Marshall ${ }^{1}$, JF Whitesides ${ }^{1}$, D Montefiori', JE Robinson ${ }^{3}$, J Kaewkungwal ${ }^{4}$,



From AIDS Vaccine 2012

Boston, MA, USA. 9-12 September 2012

\section{Background}

Antibody Dependent Cellular Cytotoxicity (ADCC) may be a contributing factor of immune responses controlling HIV-1 replication. Understanding the epitopes recognized by ADCC-mediating antibodies is likely to be important for the development of an effective AIDS vaccine. We characterized the epitope specificity and breadth of the ADCC-mediating antibody response elicited by the RV144 vaccine regimen.

\section{Methods}

Twenty-three monoclonal antibodies (mAbs) were isolated from 6 vaccine recipients either from $\mathrm{IgG}^{+}$memory B cells cultured at near clonal dilution for 14 days $(n=115,200)$ followed by sequential screenings of culture supernatants for HIV-1 gp120 Env binding, or from memory B cell $(n=206,745)$ sorting for HIV-1 group $M$ consensus gp140Con.S Env binding. Target cells infected with infectious molecular clones expressing Clade A/E (CM235), $\mathrm{B}(\mathrm{BaL})$, and $\mathrm{C}$ (DU422 and DU151) env were used to characterize the specificity and breadth of the $23 \mathrm{mAbs}$ that display ADCC activity. We defined the epitope specificity of the isolated mAbs by mapping with B.MN and/or AE.92TH023 linear peptides in ELISA and with Fabcompetition in ADCC assays.

\section{Results}

Linear mapping revealed that $2 \mathrm{mAbs}$ recognized the $\mathrm{V} 2$, and $1 \mathrm{mAb}$ the $\mathrm{V} 3$ regions of the gp120. Nineteen (19) mAbs recognized conformational epitopes overlapping the $\mathrm{C} 1 \mathrm{~A} 32$ epitope; one $\mathrm{mAb}(\mathrm{CH} 20)$ recognized a conformational epitope that was not blocked by any of the Fabs (A32, 19B, 17b) utilized in our assay. Fourteen of the 20 mAbs directed against conformational epitopes mediated ADCC against the clade B BaL Env; 4 recognized the clade C DU151 Env and 1 recognized the clade C DU422 Env.

\section{Conclusion}

The RV144 vaccine regimen induced broadly-reactive ADCC Abs that recognized both unique and overlapping regions of gp120. The mAbs with the greatest breadth may be useful for passive protection trials in rhesus macaques. If protective in non-human primates, the epitopes recognized by these mAbs may inform immunogen design.

\section{Author details}

'Duke University, Durham, NC, USA. ²University of Alabama at Birmingham, Birmingham, AL, USA. ${ }^{3}$ Tulane University School of Medicine, New Orleans, LA, USA. ${ }^{4}$ Tropical Hygiene, Mahidol University, Bangkok, Thailand. ${ }^{5}$ Armed Forces Research Institute of Medical Sciences, Bangkok, Thailand. ${ }^{6}$ Clinical Tropical Medicine, Mahidol University, Bangkok, Thailand. ${ }^{7}$ Department of Disease Control, Ministry of Public Health, Nonthaburi, Thailand. ${ }^{8}$ US Military HIV Research Program, Rockville, MD, USA.

Published: 13 September 2012

doi:10.1186/1742-4690-9-S2-039

Cite this article as: Pollara et al:: Vaccine-induced ADCC-mediating antibodies target unique and overlapping envelope epitopes. Retrovirology 2012 9(Suppl 2):039.

${ }^{1}$ Duke University, Durham, NC, USA

Full list of author information is available at the end of the article

(C) 2012 Pollara et al; licensee BioMed Central Ltd. This is an Open Access article distributed under the terms of the Creative Commons 